\title{
El ateneo de morbilidad y mortalidad como herramienta de aprendizaje colectivo en un servicio de cirugía general
}

The morbidity and mortality athenaeum as a collective learning tool in a general surgery service

\author{
Pablo Valsangiacomo, ${ }^{1}$ Leandro Telles, ${ }^{2}$ Daniel González ${ }^{3}$
}

DOI: $10.31837 /$ cir.urug/3.2.2

Recibido: 05 de febrero de 2019

Aceptado: 23 de julio de 2019

\section{Resumen}

Introducción. La enseñanza en la clínica quirúrgica representa un desafío docente ya que comprende el pregrado, el posgrado, así como a los docentes en formación. En la misma debemos de incluir aspectos docentes, asistenciales, de investigación así como de gestión clínica.

Objetivos. Analizar el ateneo de morbilidad y mortalidad como herramienta docente mediante el análisis del proceso asistencial del paciente complicado.

Método En el periodo mayo 2017 a mayo 2018 se registraron todos los pacientes complicados en la Clínica Quirúrgica 3.

Resultados Se analizaron 38 pacientes complicados de un total de 470 operados de coordinación (8\%). El tiempo al diagnóstico de la complicación fue de 4 días y la media de internación fue de 14 días. De acuerdo a Dindo - Clavien se clasificaron en los siguientes grados: I: 6, II:8, IIIa: 3, IIIb: 5, IVa: 4,IVb: 5 yV: 7 casos. La mortalidad fue del 1,5\%, vinculada a la cirugía compleja, pacientes añosos y al diagnóstico tardío de la complicación.

Conclusiones El ateneo de morbilidad y mortalidad constituye una herramienta educativa para todos los niveles formativos en la clínica quirúrgica. Conocer las complicaciones, su frecuencia, su tipo y su forma de prevenirlas nos permitió elaborar estrategias para realizar el diagnóstico precoz.

Palabras clave: ateneo, morbilidad, mortalidad,

\section{Abstract}

Introduction. Teaching in the surgical clinic represents a teaching challenge since it includes undergraduate, postgraduate, as well as training teachers. In it we must include teaching, assistance, research as well as clinical management aspects.

Objectives. To analyze the morbidity and mortality athenaeum as a teaching tool through the analysis of the complicated patient care process.

Method In the period May 2017 to May 2018, all the complicated patients were registered in the Surgical Clinic number 3.

Results 38 complicated patients from a total of 470 coordination operations (8\%) were analyzed. The time to diagnosis of the complication was 4 days and the average hospitalization time was 14 days. According to Dindo - Clavien they were classified in the following grades: I: 6, II: 8, IIIa: 3, IIIb: 5, IVa: 4, IVb: 5 and V: 7 cases. Mortality was $1.5 \%$, linked to complex surgery, elderly patients and late diagnosis of the complication.

Conclusions The morbidity and mortality athenaeum constitutes an educational tool for all training levels in the surgical clinic. Knowing the complications, their frequency, their type and how to prevent them allowed us to develop strategies to make the early diagnosis.

Keywords: athenaeum, morbidity, mortality, tool, learning.

herramienta, aprendizaje.

\footnotetext{
${ }^{1}$ Profesor Agregado Titular de Cirugía General. Clínica Quirúrgica 3. Hospital Maciel. Facultad de Medicina, Universidad de la República. Montevideo. Uruguay.pvalsangiacomo@gmail.com ORCID https://orcid.org/0000-0003-2028-5552

${ }^{2}$ Asistente de Cirugía General. Clínica Quirúrgica 3. Hospital Maciel. Facultad de Medicina, Universidad de la República. Montevideo. Uruguay. ORCID https://orcid.org/ 0000-0003-4830-5578

${ }^{3}$ Profesor Titular de Cirugía General. Clínica Quirúrgica 3. Hospital Maciel. Facultad de Medicina, Universidad de la República. Montevideo. Uruguay. ORCID https://orcid.org/0000-0003-3916-9201
} 


\section{Introducción}

La enseñanza en la clínica quirúrgica representa un desafío docente ya que comprende el pregrado, el posgrado, así como a los docentes en formación. La misma se realiza en diferentes ámbitos que incluye actividades en emergencia, en sala de internación, policlínica, así como en block quirúrgico. En ella debemos de incluir aspectos docentes, asistenciales, de investigación, así como de gestión clínica.

El ateneo de morbilidad y mortalidad constituye un ámbito de aprendizaje, de trascendental importancia, donde confluyen todos los aspectos de la enseñanza previamente mencionados. Es denominado como "la hora de oro de la educación quirúrgica”.

El análisis de las complicaciones de la cirugía general resulta de suma importancia debido a su elevada frecuencia, las mismas se encuentran relacionadas a errores médicos, pueden evitarse, implican mayores gastos en salud, se consideran indicadores de calidad asistencial y podemos actuar directamente para disminuir su incidencia y severidad. ${ }^{1}$

Este ateneo es un indicador de calidad asistencial, donde se analiza de forma constructiva y reflexiva, el proceso asistencial del paciente que presento la complicación.

El objetivo general es analizar el proceso de enseñanza de los integrantes de la Clínica Quirúrgica 3 en el ateneo de morbilidad y mortalidad.

El objetivo específico es conocer y analizar el índice de morbilidad y mortalidad, sus causas y clasificarlas; en pacientes operados de coordinación en la clínica quirúrgica 3 del Hospital Maciel.

\section{Material y método}

Se realizó un estudio retrospectivo, observacional y descriptivo.

Fueron registrados todos los pacientes presentados en el ateneo de morbi-mortalidad durante el periodo mayo 2017 - mayo 2018.

El ateneo es realizado con día y hora determinado y la participación es obligatoria para todos los integrantes de nuestro servicio. La presentación del caso complicado debe de reunión las siguientes características:

- Ser preparado y presentado por el cirujano que opero al paciente.

- Los videos de la cirugía deben presentarse sin editar.

- Debe de analizar todo el proceso asistencial del paciente.

- Presentación de 15 minutos.

En el ateneo se registran todas las complicaciones en una base de datos específica para las mismas y fueron clasificadas de acuerdo a la escala de Dindo - Clavien. ${ }^{2}$ (Tabla 1 ).

La base de datos y el análisis estadístico fue realizada mediante el programa SPSS 22, para Windows, Chicago, ILL. Fue considerado estadísticamente significativo $p<0.05$. 
Tabla 1. Clasificación de las complicaciones de Dindo - Clavien.

\begin{tabular}{|c|c|c|}
\hline \multicolumn{3}{|r|}{ Clasificación de Dindo - Clavien } \\
\hline Grado & & Definición \\
\hline \multicolumn{2}{|l|}{ I } & $\begin{array}{l}\text { Cualquier desviación del postoperatorio normal que no requiera } \\
\text { reintervención quirúrgica. Incluye antiheméticos, antipiréticos, } \\
\text { analgésicos, diuréticos, electrolitos y fisioterapia. } \\
\text { Incluye infección de la herida resuelta en la cama del paciente. }\end{array}$ \\
\hline II & & $\begin{array}{l}\text { Necesidad de tratamiento farmacológico diferente al grado I. incluye } \\
\text { transfusiones y alimentación parenteral. }\end{array}$ \\
\hline \multirow[t]{3}{*}{ III } & & Necesidad de tratamiento quirúrgico, endoscópico o percutáneo. \\
\hline & $\mathrm{a}$ & Sin anestesia general \\
\hline & $\mathrm{b}$ & Con anestesia general \\
\hline \multirow[t]{3}{*}{ IV } & & Necesidad de internación en Unidad de Terapia Intensiva \\
\hline & $\mathrm{a}$ & Falla de un órgano \\
\hline & $\mathrm{b}$ & Falla multiorgánica \\
\hline $\mathbf{V}$ & & Muerte \\
\hline
\end{tabular}

\section{Resultados}

En el período analizado fueron registrados 38 pacientes complicados de un total de 470 pacientes operados (8\%). Edad promedio fue de 52 años. 22 pacientes fueron del sexo femenino (58\%).

El tiempo medio al diagnóstico de la complicación fue de 4 días (0 - 23 días). La estadía hospitalaria promedio fue de 14 días (1 a 48 días).

De acuerdo a la clasificación de Dindo-Clavien se distribuyeron de la siguiente manera:

(Tabla 2 y gráfico 1).

Tabla 2. Clasificación de Distribución de complicaciones de acuerdo a clasificación de Dindo-Clavien

\begin{tabular}{ccc}
\hline Dindo-Clavien & casos & $\mathbf{\%}$ \\
\hline I & 6 & 16 \\
II & 8 & 21 \\
IIIa & 3 & 8 \\
IIIb & 5 & 13 \\
IVa & 4 & 11 \\
IVb & 5 & 13 \\
\hline
\end{tabular}




\begin{tabular}{ccc}
\hline $\mathbf{V}$ & 7 & 18 \\
Total & 38 & 100 \\
\hline
\end{tabular}

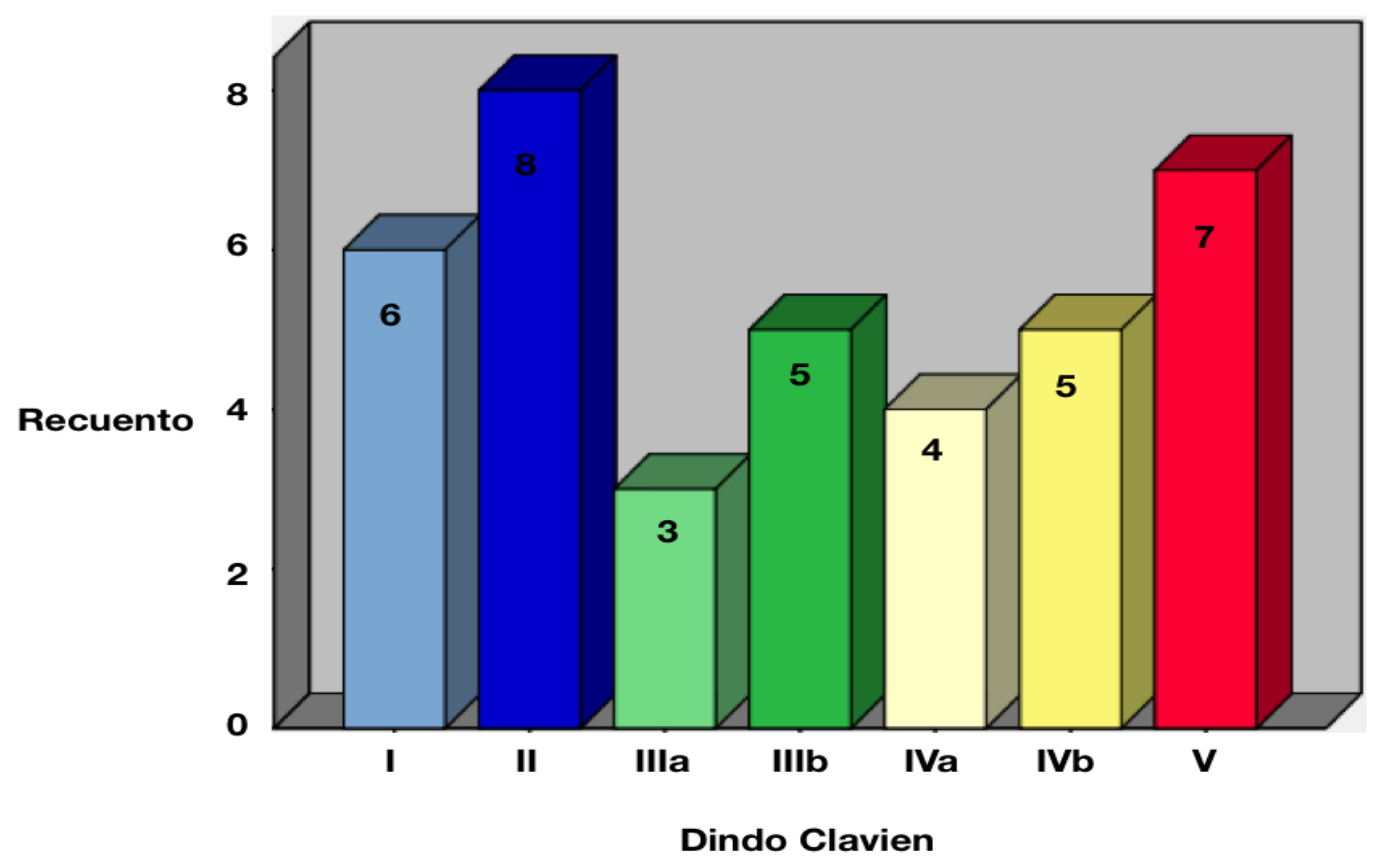

Gráfico 1. Clasificación de Dindo - Clavien.

La mortalidad fue del 1,5\% (7/470). La misma se presentó en pacientes añosos ( $>65$ años) $p<0.05$, luego de cirugías complejas y vinculadas a falla de sutura digestiva.(Tabla 3).

Tabla 3. Análisis de la mortalidad. Patología y causa.

\begin{tabular}{|c|c|}
\hline PATOLOGIA & CAUSA \\
\hline DPC. & Infarto mesenterico. \\
\hline Reconstruccion TD. & Falla sutura esofago-gastrica. \\
\hline T. Klastkin. & Sepsis biliar. Drenaje percutaneo. \\
\hline Hepatectomia. & Perforacion colon mas lesion VBP. \\
\hline Recto. & Hemoperitoneo mas falla sutura. \\
\hline Biopsia hepatica. & Hemoperitoneo masivo. \\
\hline Carcinomatosis peritoneal. & $\begin{array}{l}\text { Absceso pelviano. Aspergilosis } \\
\text { pulmonar. Sepsis. }\end{array}$ \\
\hline
\end{tabular}


Con respecto al manejo perioperatorio, el mismo fue adecuado, si bien debemos destacar una subestimación en los síntomas de alarma en el paciente complicado, lo cual puede determinar un retardo en el diagnóstico de la misma.

\section{Discusión}

El análisis de las complicaciones en un servicio de cirugía general representa un desafío docente y asistencial, que comprende a todos los integrantes del servicio, el cual debe de realizarse en un ámbito apropiado y predeterminado como lo es el ateneo de morbilidad y mortalidad. Este nos brinda la posibilidad de desarrollar aspectos educativos, asistenciales de investigación así como de gestión clínica.

En el mismo se realiza una auditoria de todo el proceso asistencial del paciente complicado, mediante un análisis crítico, reflexivo y constructivo.

La participación debe incluir al pregrado (estudiantes, pasantes y practicantes internos), a los residentes así como todo el staff docente.

A su vez creemos de suma importancia que participen todas las otras especialidades médicas que participaron en la asistencia del paciente complicado.

El ateneo debe de ser coordinado por un docente referente del servicio quien será que dirija el mismo y genere un ámbito de aprendizaje positivo que estimule la participación activa de todos los integrantes. (Estructura horizontal de discusión). ${ }^{3}$

Los centros académicos en la actualidad presentan un gran desafío en la enseñanza; donde los requerimientos educativos son cada vez mayores y el tiempo disponible para la educación es cada vez menor. Por lo cual el ateneo de morbilidad y mortalidad representa una excelente oportunidad de aprendizaje colectivo, con la inversión de una hora semanal, con el objetivo de evaluar la etiopatogenia, el diagnostico, el tratamiento y la prevención de las complicaciones. Frente a estas limitaciones debemos de asegurar una adecuada calidad del ateneo.

Además del aspecto educativo, el ateneo nos permite una medición de calidad asistencial, ya que la tasa de efectos negativos generados por nuestra práctica quirúrgica es un índice de calidad de la medicina que estamos brindando.

Gore $^{4}$ en el año 2006, evaluando 34instituciones de Estados Unidos y Canadá donde se desarrollaban ateneos de morbimortalidad estructurados, resalto el valor no solo educativo sino como una herramienta efectiva para reducir errores futuros en cirugía. 
El ateneo presenta objetivos precisos y condiciones claras para favorecer su desarrollo. (Tabla 4).

Ateneo de morbilidad y mortalidad

Tabla 4. Características del ateneo de morbilidad y mortalidad

\section{Objetivos:}

- Aprender de errores y complicaciones.

- Modificar comportamientos y criterios sobre la base de experiencias propias.

- Prevenir la repetición de errores que conlleven complicaciones.

- Establecer una herramienta educativa valida que promueva mejoras en la calidad de atención médica.

\section{Condiciones:}

- Periodicidad semanal.

- Ambiente positivo, de crítica constructiva y no de castigo y culpa.

- Brevedad en la presentación de los casos.

- Registrar todas las complicaciones y clasificarlas.

- $\quad$ No preseleccionar los casos

- Presentación de casos recientes, lo que estimula la discusión activa.

El índice de complicaciones en nuestro servicio se encuentra dentro de parámetros internacionales ya que es considerado aceptable entre un 6\% y $11 \%$ en cirugía de coordinación. ${ }^{5,6}$ Si bien esta cifra depende de la patología, terreno del paciente, tipo de cirugía y su oportunidad.

En cuanto a la mortalidad, en nuestro servicio se encuentra vinculada a procedimientos complejos en pacientes añosos y a complicaciones infecciosas.

Los diferentes integrantes del servicio han desarrollado varias líneas de investigación a partir de problemas que se presentan con el diagnóstico y tratamiento de las complicaciones.

Los estudiantes han realizado su monografía en el curso de cirugía mediante el análisis de las complicaciones en el año 2017.

Con respecto al manejo perioperatorio, el mismo fue adecuado, si bien debemos destacar una subestimación en los síntomas de alarma en el paciente complicado, lo cual puede determinar un retardo en el diagnóstico de la misma.

A su vez los residentes y docentes de nuestro servicio realizaron un estudio de investigación para detectar precozmente la complicación mediante la utilización de marcadores humorales de respuesta inflamatoria (PCR y procalcitonina). ${ }^{8}$ 
Un aspecto muy importante que nos permite el ateneo es el análisis de la gestión asistencial. Debemos de tener en cuenta que la presencia de alguna complicación aumenta significativamente los costos en salud. En nuestro trabajo la estadía hospitalaria se triplicó en el paciente que presento la complicación, lo cual coincide con múltiples trabajos publicados al respecto. ${ }^{6,9,10}$ A esto debemos de sumarle los costos del mayor número de estudios solicitados, reintervenciones así como de ingreso en cuidados intensivos.

\section{Conclusiones}

El ateneo de morbilidad y mortalidad constituye una herramienta educativa para todos los niveles formativos de nuestra Facultad.

Las complicaciones presentaron una frecuencia comparablecon cifras internacionales. El conocimiento de nuestros índices de morbilidad, mortalidad, su tipo y mecanismo de producción nos permitió elaborar estrategias para el diagnóstico precoz. A su vez es un indicador de calidad asistencial, así como de gestión en salud.

\section{Bibliografía}

1- Pekolj J, Ardiles V, Hyon SH. Complicaciones de la cirugía abdominal. $1^{\text {a }}$ Ed .Buenos Aires. Delhospital ediciones, 2015.

2- Dindo D, Demartines N, Clavien PA. Classification of surgical complications: a new proposal with evaluation in a cohort of 6336 patients and results of a survey. Ann Surg. 2004;240:205-13.

3- Algieri RD, Grzona E, Ferrante MS, Franco Alanis F, Cipollone S. Valoración del ateneo de morbimortalidad como herramienta de enseñanza durante la residencia de cirugía general. HospAeronáut Cent. 2014; 9:92-101.

4- Gore DC. National survey of surgical morbidity and mortality conferences. Am J Surg. 2006;191:708-714.

5- Tevis SE, Cobian AG, Truong HP, Craven MW, Kennedy GD. Implications of multiple complications on the postoperative recovery of general surgery patients. Ann Surg. 2016;263:1213-8.

6- Gomez-Rosado JC, Salas-Turrens J, Olry-de-Labry-Lima A. Análisis de los costes económicos asociados a las complicaciones en cirugia general. Cir Esp. 2018;96:292-9.

7- Gargiullo V, Núñez V, Osterkamp K.,Patri F, Perrone E, Quagliata, V.Complicaciones postquirúrgicas en cirugías de coordinación[monografía de grado]. Montevideo: Clínica Quirúrgica 3, Hospital Maciel,Facultad de Medicina, UDELAR; set. 2017.

8- Wagner G, ValsangiacomoP,Rodriguez Cantera G, Segura D, Ruso L. Procalcitonina y proteína $\mathrm{C}$ reactiva como marcadores precoces de falla de sutura digestiva. Presentado En: 68 Congreso Uruguayo de Cirugía. 2017. 28-30 Noviembre, Montevideo. Uruguay. 
9- Eappen S, Lane BH, Rosenberg B, Lipsitz SA, Sadoff D, Matheson D, et al. Relationship between occurrence of surgical complications and hospital finances. JAMA. 2013;309:1599-606.

10- Dimick JB, Pronovost PJ, Cowan JA, Lipsett PA. Complications and costs after high-risk surgery: Where should we focus quality improvement initiatives? J Am CollSurg. 2003;196:671-8. 